\title{
The Reduced Basis Method Applied to Transport Equations of a Lithium-Ion Battery
}

\author{
Stefan Volkwein ${ }^{*}$ Andrea Wesche ${ }^{*} \dagger$ \\ ${ }^{*}$ Universität Konstanz, Fachbereich Mathematik und Statistik, Universitätsstraße 10, D-78457 Konstanz, \\ E-mail: stefan.volkwein@uni.konstanz.de \\ $\dagger$ Adam Opel AG, Bahnhofsplatz, D-65423 Rüsselsheim, E-mail: Andrea.Wesche@de.opel.com
}

\begin{abstract}
In this paper we consider a coupled system of nonlinear parametrized partial differential equations $\left(\mathrm{P}^{2} \mathrm{DEs}\right)$, which models the concentrations and the potentials in lithium-ion batteries. The goal is to develop an efficient reduced basis approach for the fast and robust numerical solution of the $\mathrm{P}^{2} \mathrm{DE}$ system. Numerical examples illustrate the efficiency of the proposed approach.
\end{abstract}

Index Terms-finite volume method, greedy algorithm, lithium-ion battery, reduced basis method

\section{INTRODUCTION}

The modelling of lithium-ion batteries has received an increasing amount of attention in the recent past. Several companies worldwide are developing such batteries for consumer electronic applications, in particular, for electric-vehicle applications. To achieve the performance and lifetime demands in this area, exact mathematical models of the battery are required. Moreover, the multiple evaluation of the battery model for different parameter settings involves a large amount of time and experimental effort. Here, the derivation of reliable mathematical models and their efficient numerical realization are very important issues in order to reduce both time and cost in the improvement of the performance of batteries.

In the present work we consider a mathematical model for lithium-ion batteries which describes the transport processes by a partial differential equation system. This model is developed in the paper by Popov et al. [17]. The physical and chemical details can be found in [13] and [14]. The equation system models a physico-chemical micro-heterogeneous battery model.

We discretize this by the finite volume method and the backward Euler method. The reduced basis methodology for by finite volumes discretized systems can be found in [10]. The discretized model is reduced by the reduced basis method [16]. Our numerical tests will illustrate the efficiency of this approach.

A popular battery model is the one developed by Newman [4], [15], which was implemented and tested [5]. Let us also refer to the work [6], where a different battery model is derived. For an equation system which describes a physico-chemical macro-homogeneous battery model the well posedness is shown by $\mathrm{Wu}$ et al. [19].

\section{BATTERY MODEL}

\section{PDE Model}

Let $\Omega \subset \mathbb{R}$ be an open interval, which is divided in three disjunct open sub-intervals $\Omega_{c}, \Omega_{e}, \Omega_{a} \subset \mathbb{R}$, see Figure 2.1. For $t_{\text {end }}>0$ we define $Q:=\Omega \times\left(0, t_{\text {end }}\right)$ and let $c, \phi: Q \rightarrow \mathbb{R}$ and $\alpha, \beta, \lambda, \kappa: \mathbb{R}^{2} \rightarrow \mathbb{R}$, the notations

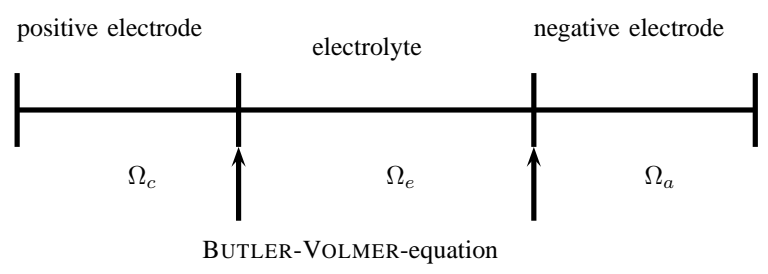

Fig. 2.1. Structure of the considered battery domain

can be found in Table 1.2 in the appendix. The transport processes in a battery, i.e. transport of mass and charge, are described by the equations [17]

$$
\begin{array}{r}
\frac{\partial c}{\partial t}-\nabla \cdot(\alpha(c, \phi) \nabla c+\beta(c, \phi) \nabla \phi)=0 \\
-\nabla \cdot(\lambda(c, \phi) \nabla c+\kappa(c, \phi) \nabla \phi)=0
\end{array}
$$

in $\Omega_{c} \times\left(0, t_{\mathrm{end}}\right), \Omega_{e} \times\left(0, t_{\mathrm{end}}\right)$ and $\Omega_{a} \times\left(0, t_{\mathrm{end}}\right)$, where " $\nabla$ " denotes the gradient and " $\nabla \cdot$ " the divergence.

The positive electrode is $\Omega_{c}$ (cathode for discharge), the electrolyte $\Omega_{e}$ and the anode $\Omega_{a}$ (anode for discharge).

\section{Boundary/Interface Conditions}

The boundary conditions are

$$
\begin{array}{lll}
\frac{\partial c}{\partial \nu}=0, & \phi=0 & \text { on }\left(\partial \Omega \cap \partial \Omega_{c}\right) \times\left(0, t_{\mathrm{end}}\right) \\
\frac{\partial c}{\partial \nu}=0, & \frac{\partial \phi}{\partial \nu}=-\frac{I}{\sigma_{a}} & \text { on }\left(\partial \Omega \cap \partial \Omega_{a}\right) \times\left(0, t_{\mathrm{end}}\right)
\end{array}
$$

where $\nu$ is the outer unit normal vector, $I: \mathbb{R}^{+} \rightarrow \mathbb{R}$ (time-dependent current) and $\sigma_{a} \in \mathbb{R}^{+} \backslash\{0\}$ (electric conductivity multiplied with the cross section). The initial condition is given by

$$
c\left(x, t_{0}=0\right)=c_{0}(x), x \in \Omega
$$


The interface conditions are given by

$$
\begin{aligned}
& -(\alpha(c, \phi) \nabla c+\beta(c, \phi) \nabla \phi) \\
= & \left\{\begin{array}{l}
\left.\mathcal{I}\left(c_{e c}, c_{c}, \phi_{e c}, \phi_{c}\right)\right|_{\left(0, t_{e n d}\right)} \text { in }\left(\partial \Omega_{c} \cap \partial \Omega_{e}\right) \\
-\left.\mathcal{I}\left(c_{e a}, c_{a}, \phi_{e a}, \phi_{a}\right)\right|_{\left(0, t_{e n d}\right)} \text { in }\left(\partial \Omega_{e} \cap \partial \Omega_{a}\right)
\end{array}\right. \\
& -(\lambda(c, \phi) \nabla c+\kappa(c, \phi) \nabla \phi) \\
= & \left\{\begin{array}{l}
\left.\mathcal{J}\left(c_{e c}, c_{c}, \phi_{e c}, \phi_{c}\right)\right|_{\left(0, t_{e n d}\right)} \text { in }\left(\partial \Omega_{c} \cap \partial \Omega_{e}\right) \\
-\left.\mathcal{J}\left(c_{e a}, c_{a}, \phi_{e a}, \phi_{a}\right)\right|_{\left(0, t_{e n d}\right)} \text { in }\left(\partial \Omega_{e} \cap \partial \Omega_{a}\right)
\end{array}\right.
\end{aligned}
$$

where $c_{e a}$ is the concentration in the electrolyte at the negative electrode interface:

$$
c_{e a}(t)=\lim _{h \rightarrow 0} c\left(\left.x\right|_{\Omega_{e} \cap \Omega_{a}}-h, t\right)
$$

and $h>0$ is small enough, i.e. $\left.x\right|_{\Omega_{e} \cap \Omega_{a}}-h \in \Omega_{e}$. Analogously

$$
\begin{aligned}
c_{e c}(t) & =\lim _{h \rightarrow 0} c\left(\left.x\right|_{\Omega_{e} \cap \Omega_{c}}+h, t\right) \\
c_{c}(t) & =\lim _{h \rightarrow 0} c\left(\left.x\right|_{\Omega_{e} \cap \Omega_{c}}-h, t\right) \\
c_{a}(t) & =\lim _{h \rightarrow 0} c\left(\left.x\right|_{\Omega_{e} \cap \Omega_{a}}+h, t\right)
\end{aligned}
$$

for sufficient small $h>0$. The variables $\phi_{c}, \phi_{a}, \phi_{e c}, \phi_{e a}$ are defined in the same way. We write $c_{s}$ for the concentration in the solid part, i.e. in the negative and positive electrode, and $c_{e}$ for the concentration in the electrolyte, $\phi_{s}$ and $\phi_{e}$ are analogously denoted. The scalar functions $\mathcal{I}: \mathbb{R}^{4} \rightarrow \mathbb{R}$ and $\mathcal{J}: \mathbb{R}^{4} \rightarrow \mathbb{R}$ are defined by

$$
\begin{aligned}
\mathcal{I}\left(c_{e}, c_{s}, \phi_{e}, \phi_{s}\right)= & \frac{\mathcal{J}\left(c_{e}, c_{s}, \phi_{e}, \phi_{s}\right)}{F} \\
\mathcal{J}\left(c_{e}, c_{s}, \phi_{e}, \phi_{s}\right)= & k \sqrt{\frac{c_{e}}{c_{e}^{0}}} \sqrt{\frac{c_{s}}{c_{s}^{0}}} \sqrt{1-\frac{c_{s}}{c_{s, \max }}} \\
& \cdot 2 \sinh \left(\frac{F}{2 R T}\left(\phi_{s}-\phi_{e}-U_{0}\left(c_{s}\right)\right)\right)
\end{aligned}
$$

where $F=96486 \frac{\mathrm{A} \cdot \mathrm{s}}{\mathrm{mol}}$ is the Faraday constant, $R=$ $8.314 \frac{A \cdot V \cdot s}{K \cdot m o l}$ is the gas constant and $T>0[K]$ is the temperature. The function $U_{0}: \mathbb{R} \rightarrow \mathbb{R}$ is the over potential and depends on the concentration $c$ in the electrodes. The coefficient functions are defined as

$$
\begin{array}{lll}
\alpha(c, \phi):=D_{e}(c, \phi)+\frac{R T}{F^{2}} \frac{\left(t_{+}(c, \phi)\right)^{2} \kappa(c, \phi)}{c} & {\left[\frac{\mathrm{cm}^{2}}{s}\right]} \\
\beta(c, \phi):=\kappa(c, \phi) \frac{t_{+}(c, \phi)}{F} & {\left[\frac{\mathrm{mol}}{V \cdot c m \cdot s}\right]} \\
\lambda(c, \phi):=\frac{R T}{F} \frac{t_{+}(c, \phi) \kappa(c, \phi)}{c} & {\left[\frac{A \cdot c m^{2}}{m o l}\right]}
\end{array}
$$

where the transference number $t_{+}$is zero in the electrodes and larger than zero in the electrolyte and $\kappa$ is the ionic/electric conductivity; $\kappa, t_{+}, D_{e}: \mathbb{R}^{2} \rightarrow \mathbb{R}$. To measure the battery parameters experimentally it is assumed, that they are polynomials in $c$ and $\phi$.

The homogeneous Neumann boundary conditions for the concentration (2.2) mean that no flux of lithium(-ions) can pass through. The inhomogeneous Neumann boundary condition for the potential is Ohm's law, the homogeneous Dirichlet boundary condition have no physical meaning. It ensures the uniqueness of the solution if one exists.

The interface conditions describe the exchange of the lithium-ions at the interfaces which are modeled by the Butler-Volmer-equation [1].

For physical reasons we assume that

$$
c(x, t) \geq 0 \forall x \in \Omega, t \in\left(0, t_{\text {end }}\right)
$$

We remark that the coefficient functions $\alpha$ and $\kappa$ are larger than zero for physical reasons: the diffusivity $D_{e}$ and the conductivity $\kappa$ are larger than zero. Because of the definition of the transference number $t_{+}$the coefficient functions $\beta$ and $\lambda$ are equal or larger than zero.

\section{Discretization of the Problem}

We discretize the partial differential equation system (2.1a)-(2.1b) with the appropriate boundary (2.2) and interface conditions (2.4) by the cell centered finite volume method. We divide therefore $\Omega_{c}$ in $N_{c} \in \mathbb{N}, \Omega_{e}$ in $N_{e} \in \mathbb{N}$ and $\Omega_{a}$ in $N_{a} \in \mathbb{N}, N_{D}=N_{c}+N_{e}+N_{a}$, equidistant control volumes of the width $\Delta x$. We use the method of lines and solve the equation system for every time step. The time step size is $\Delta t$. The integrals over the spatial we approximate by the middle point rule, the integrals over the time by the backward Euler method, for details cf. [17]. These discretized equations are implemented in MATLAB 7.10.0 (R2010a).

\section{REDUCED BASIS METHOD}

\section{Initial Point}

We consider a parametrized PDE which we want to solve for many parameter sets, e.g. for parametric studies. The better the numerical model approximates the physical phenomenon, the more expensive the computation gets. So in some cases e.g. a parameter analysis needs too much effort, because a single computation is too expensive. Therefore one has to develop a reduced model to get cheap solutions.

The reduced basis method is based on the discretized model: the idea is to compute a "few" times an expensive solution to different parameter sets which are in the range of interest. With the knowledge of these so called "true" solutions basis vectors are computed. The approach is that the reduced solutions in the parameter set of interest are linear combinations of these basis vectors.

An assumption is that the error between the "exact" analytical solution and the "true" numerical solution is small in contrast to the error between the "true" and the "reduced" solution.

A big advantage of the present method is that you determine the error between the true and reduced solution during "developing" your reduced model ( $\rightarrow$ Greedy algorithm). A further property is that the method has two phases: the offline computation in which the reduced 
model is set which fulfills the given error tolerance and in which the needed true solutions are computed and the online phase in which the reduced solution(s) are computed. The offline part is expensive and the online phase is cheap.

\section{Approach}

In the following we have to resolve how to choose the true solution and how to estimate the error between the reduced and true solution. The (POD-)Greedy algorithm ensures both issues.

We now describe how to apply the reduced basis method on our battery model. The transport equations of the battery (2.1a)-(2.1b) depend on many parameters: on geometrical parameters (e.g. the width of the electrode) on state parameters (e.g. temperature) and on battery parameters (e.g. the diffusion coefficient). We note these parameters with $\mu \in \mathcal{D}$ and assume that all these different parameters are polynomials in $c$ and $\phi$, some are of course constant and so polynomials of the degree zero.

We write $u^{\mathcal{N}} \in \mathcal{X}^{\mathcal{N}}$ for a piecewise linear functions, its coefficient are denoted with $\underline{u}^{\mathcal{N}} \in \mathbb{R}^{N}$. The discretized problem is now the following: Find a $u^{\mathcal{N}} \in \mathcal{X}^{\mathcal{N}}$ so that

$$
\begin{aligned}
F^{\mathcal{N}}\left(\underline{u}^{\mathcal{N}} ; \mu\right) & =0 \\
\Leftrightarrow\left\langle F^{\mathcal{N}}\left(\underline{u}^{N} ; \mu\right), \underline{v}^{\mathcal{N}}\right\rangle_{W} & =0 \quad \forall v^{\mathcal{N}} \in \mathcal{X}^{\mathcal{N}}
\end{aligned}
$$

where the mass matrix $W$ is in the present case given by $W=\Delta x \cdot \mathbb{1} \in \mathbb{R}^{\mathcal{N} \times \mathcal{N}}$.

We approximate the finite volume space by a $N$ dimensional space $\mathcal{X}^{N}$ which is spanned by "snapshots". A snapshot is a true solution to a specific parameter set $\mu \in \mathcal{D}$ and time node $t \in\left(0, t_{\text {end }}\right)$. We assume now, that we have a (orthonormalized) basis $\Xi=\left(\xi_{1}, \ldots, \xi_{N}\right) \epsilon$ $\mathbb{R}^{\mathcal{N} \times N}$ of this space and that the reduced solution can be written as

$$
\underline{u}^{N}(\mu)=\sum_{j=1}^{N} \theta_{j}^{u^{N}}(\mu) \xi_{j}
$$

If we replace $\underline{u}^{\mathcal{N}}$ in equation (3.1) by $\underline{u}^{N}$ of equation (3.2) and choose $\underline{v}^{\mathcal{N}}=\xi_{i} \in \mathbb{R}^{\mathcal{N}}$ we get:

$$
\begin{aligned}
\Xi^{T} \cdot W \cdot F^{\mathcal{N}}\left(\underline{\theta}^{u^{N}}(\mu) \cdot \Xi ; \mu\right) & =: F^{N}\left(\theta^{u^{N}}(\mu)\right) \\
& =0
\end{aligned}
$$

The start vector for the coefficient vector for Newton's method one can get by

$$
\underline{u}_{\text {coeff }}^{\text {start }}(\mu)=\Xi^{T} \cdot W \cdot \underline{u}^{\mathcal{N}}\left(\cdot, t_{0}=0 ; \mu\right)
$$

To find a basis we use for our time dependent problem the POD-Greedy algorithm, cf. algorithm 1 and [10]. It consists of two loops: the outer loop is the "standard" Greedy, cf. for instance [16], which finds the new parameter set to which the error between the reduced and true solution is the largest. For this we need a problem specific error estimator; an error estimator for a linear by finite volumes discretized problem can be found in [10]. The inner loop reduces the trajectory $u^{\mathcal{N}}\left(\cdot, t_{n} ; \mu^{*}\right)$ in time with the POD (proper orthogonal decomposition) algorithm. This algorithm returns for each snapshot matrix $u^{\mathcal{N}}(\mu) \ell \in \mathbb{N}$ eigen-/basis vectors. One can state the number of basis vectors or the projection error of the POD method. For details to the POD algorithm cf. for instance [11], [18].

Usually we take an error estimator to estimate the error between the true and reduced solution to each parameter of the discretized parameter set. Until now we have no error estimator for the present problem, so we compare the true solution to a parameter set of the training set, to the reduced solution computed by the so far reduced basis vectors. The function $u_{R B}^{(i)}\left(\mu_{j}\right)$ is the reduced solution constructed by $i$ basis functions evaluated at the parameter set $\mu_{j} \in \mathcal{D}_{\text {train }} \subset \mathcal{D}$.

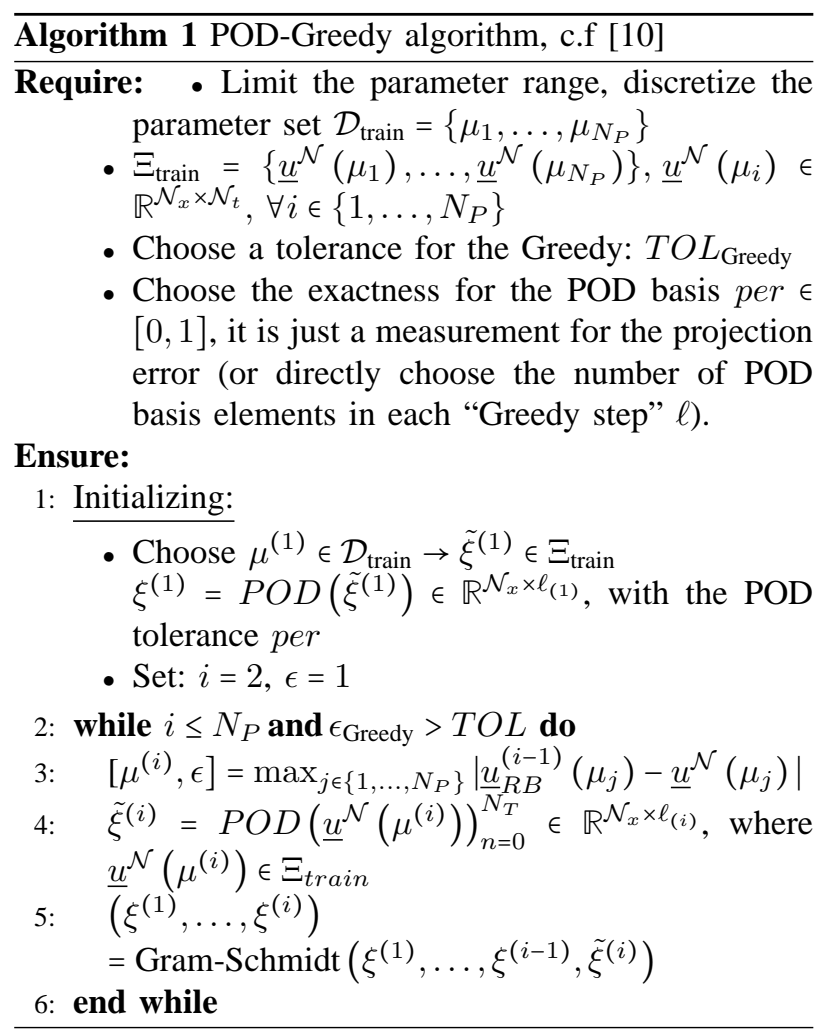

An essential property of the reduced basis method is that you can decompose the computation into an offline and online phase.

Offline: After determination of the set of parameter sets and the accuracy of the reduced solutions, we start the Greedy algorithm to compute the basis vectors, the true solutions to the chosen parameters respectively. The offline phase is computationally expensive and so the reduced basis method is only worth if you want to solve the equation system many times.

Online: In the present case we have to compute the 
coefficient vector for the basis to the different parameter sets. We get it by the damped Newton's method. This phase is cheap.

The big advantage of the reduced solution is that you know how good your reduced solution approximates the true solution. A big disadvantage is, that if you change your parameter ranges you usually have to do the expensive offline computation again.

\section{RBM applied on the battery model}

In the following we explain how to apply the reduced basis method on our discretized problem (3.1).

We denote by $F_{1}^{\mathcal{N}}(C, \Phi): \mathbb{R}^{\mathcal{N}} \times \mathbb{R}^{\mathcal{N}} \rightarrow \mathbb{R}^{\mathcal{N}}$ equation (2.1a) with boundary and interface conditions discretized by the finite volume method, analogously $F_{2}^{\mathcal{N}}(C, \Phi)$ : $\mathbb{R}^{\mathcal{N}} \times \mathbb{R}^{\mathcal{N}} \rightarrow \mathbb{R}^{\mathcal{N}}$ stands for the finite volume discretized equation (2.1b) with the boundary and interface conditions.

We choose a parameter training set for $C$ and $\Phi^{1}$ :

$$
\begin{aligned}
& \Xi_{\text {train }}^{C}=\left(c^{\mathcal{N}}\left(\mu_{1}\right), \ldots, c^{\mathcal{N}}\left(\mu_{N_{P}}\right)\right) \\
& \Xi_{\text {train }}^{\Phi}=\left(\phi^{\mathcal{N}}\left(\mu_{1}\right), \ldots, \phi^{\mathcal{N}}\left(\mu_{N_{P}}\right)\right)
\end{aligned}
$$

Let us assume that we have a basis matrix for $C$ $\Psi_{C}=\left(\psi_{1}^{c}, \ldots, \psi_{N_{B_{C}}}^{c}\right) \in \mathbb{R}^{N_{D} \times N_{B_{c}}}$ and for $\Phi \Psi_{\Phi}=$ $\left(\psi_{1}^{\phi}, \ldots, \psi_{N_{B_{\phi}}}^{\phi}\right) \in \mathbb{R}^{N_{D} \times N_{B_{\phi}}}$ then the reduced models, reduced functions respectively, are given by

$$
\begin{aligned}
& \left(\Psi^{c}\right)^{T} \cdot W \cdot\left(F_{1}^{\mathcal{N}}\left(\Psi^{c} C_{\text {coeff }}, \Psi^{\phi} \Phi_{\text {coeff }}\right)\right) \stackrel{!}{=} 0 \\
& \left(\Psi^{\phi}\right)^{T} \cdot W \cdot\left(F_{2}^{\mathcal{N}}\left(\Psi^{c} C_{\text {coeff }}, \Psi^{\phi} \Phi_{\text {coeff }}\right)\right) \stackrel{!}{=} 0
\end{aligned}
$$

We should add that the snapshots for $\Psi^{c}$ and $\Psi^{\phi}$ are taken at the same parameter sets (outer POD-Greedy), but the number of the POD basis elements could differ to achieve the same accuracy (inner POD-Greedy).

A further issue is how to choose the next parameter in the (POD-)Greedy algorithm. The error estimation we have to do for two functions $c$ and $\phi$. So we usually get two different parameter values $\mu_{c}$ and $\mu_{\phi}$, where the $\mathcal{L}^{2}$-errors of the concentration and of the potential, respectively, attain their maximum values. Then, we choose the parameter $\mu \in\left\{\mu_{c}, \mu_{\phi}\right\}$ corresponding to the greater $\mathcal{L}^{2}$-error of both.

\section{NUMERICAL EXPERIMENTS}

In this section we apply the reduced basis method to the discretized equations describing the transport processes in a lithium-ion battery (3.1). The step size in spatial is $\Delta x=1 \mu \mathrm{m}$, the time step size $\Delta t=5 \mathrm{~s}$ and we compute $N_{t}=10$ time steps. The Newton tolerance to compute the true solution is set $10^{-6}$ relatively and $10^{-9}$ absolutely. The discretization error of the finite volume solution is $\epsilon_{F V M}=\mathcal{O}\left(\frac{1}{100}\right)$.

\footnotetext{
${ }^{1}$ One can also choose different training set, e.g. cf. [7]
}

\begin{tabular}{|c|c|c|c|}
\hline & pos. electrode & electrolyte & neg. electrode \\
\hline$D_{e},\left[\frac{\mathrm{cm}^{2}}{\mathrm{~s}}\right]$ & $1.0 \cdot 10^{-9}$ & $7.5 \cdot 10^{-7}$ & $3.9 \cdot 10^{-10}$ \\
$\kappa,\left[\frac{A^{\circ \cdot c m}}{V \cdot c m}\right.$ & 0.038 & 0.002 & 1.0 \\
$c^{0},\left[\frac{\mathrm{mol}}{\mathrm{cm}}\right]$ & 0.020574 & 0.001 & 0.002639 \\
$c_{\max },\left[\frac{\mathrm{mol}}{\mathrm{cm}}\right]$ & 0.02286 & & 0.02639 \\
$U_{0},[\mathrm{~V}]$ & 0.001 & & 0 \\
$t_{+},[-]$ & 0 & 0.2 & 0 \\
$k,\left[\frac{A}{\mathrm{~cm}^{2}}\right]$ & $1.3716 \cdot 10^{-4}$ & & $5.2780 \cdot 10^{-7}$ \\
$N .,[-]$ & 10 & 30 & 10 \\
$A .,\left[\mathrm{cm}^{2}\right]$ & $\left(50 \cdot 10^{-4}\right)^{2}$ & & $\left(50 \cdot 10^{-4}\right)^{2}$ \\
\hline
\end{tabular}

TABLE 4.1

BATTERY PARAMETERS, [17]

The Newton tolerance for the reduced solution is $10^{-5}$. So the $\mathcal{L}^{2}$-error between the finite volume and reduced solution is at best less than

$$
\epsilon_{\mathcal{L}^{2}}=10^{-5} \cdot N_{x} \cdot N_{t}=0.005=: \epsilon_{\text {Greedy }}
$$

The tolerance of the POD method is set $99 \%$, cf. for the POD method for instance [18].

Our "standard" battery parameter set is listed in table 4.1, notations can be found in Table 1.2 in the appendix. We charge the battery with $1.5913 \cdot 10^{-8} A$ which corresponds to $1 C$-rate and set the temperature $T=300 K$.

\section{Test 1}

In this subsection we variate the open circuit voltage in the positive electrode: $\mu=U_{c} \in[0.001,4.501]$. We discretize this parameter set with the equidistant step width $\Delta U_{c}=0.1 \mathrm{~V}$. So we have a 46 -dimensional training set for the parameter. All the other parameters are fixed, cf. Table 4.1.

In Figure 4.1 and 4.2 the finite volumes solutions chosen by the first two iterations of algorithm 1 are presented. The associated parameters are $U_{c}=3.001 \mathrm{~V}$ and $U_{c}=4.501 \mathrm{~V}$. The concentration seems to be less sensitive to the circuit voltage than the electrical potential. The $\mathcal{L}^{2}$-error for the concentration becomes worse after the second Greedy step, but already after the first Greedy step the error is smaller than the $\mathcal{L}^{2}$-error tolerance $\epsilon_{\mathcal{L}^{2}}$ and stays smaller; the $\mathcal{L}^{2}$-error for the electrical potential gets denotative smaller with the information of a second true solution, cf. Figure 4.3 and 4.4. The same observation can be done for the $\mathcal{L}^{\infty}$-error which is not presented here. The basis functions are shown in Figure 4.5: they have the same "structure" as the finite volume solutions for one fixed time step and there is just one basis function for each Greedy step.

The speed up of the reduced solution in comparison to the true solution is 17.54 .

\section{Test 2}

In this section we variate a few parameters:

$$
\mu=\left\{D_{e_{c}}, D_{e_{e}}, D_{e_{a}}, t_{+}, k_{c}, k_{a}\right\}
$$

The subscript $c$ denotes the parameter in the positive electrode, $e$ in the electrolyte and $a$ in the 


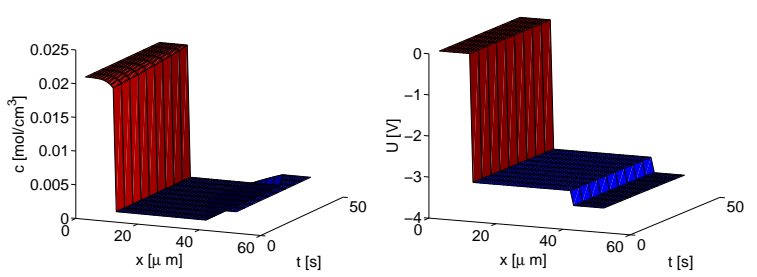

Fig. 4.1. Test 1: Finite volume solution fort the concentration (left) and the potential (right) for $U_{c}=3.001 \mathrm{~V}$
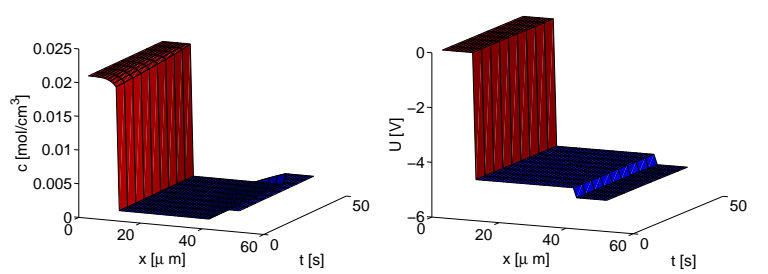

Fig. 4.2. Test 1: Finite volume solution fort the concentration (left) and the potential (right) for $U_{c}=4.501 \mathrm{~V}$
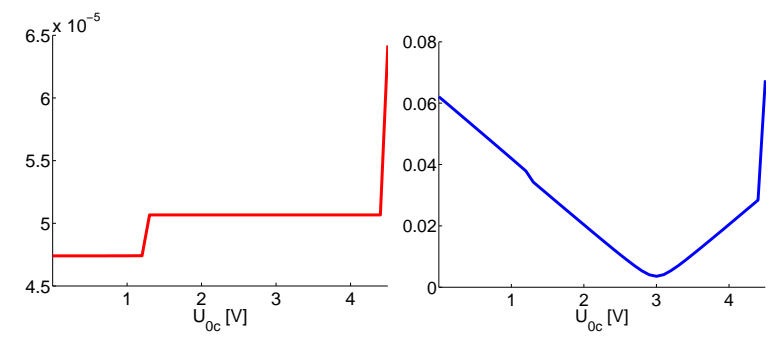

Fig. 4.3. Test 1: $\mathcal{L}^{2}$-error after the first Greedy step for the concentration (left) and the potential (right).

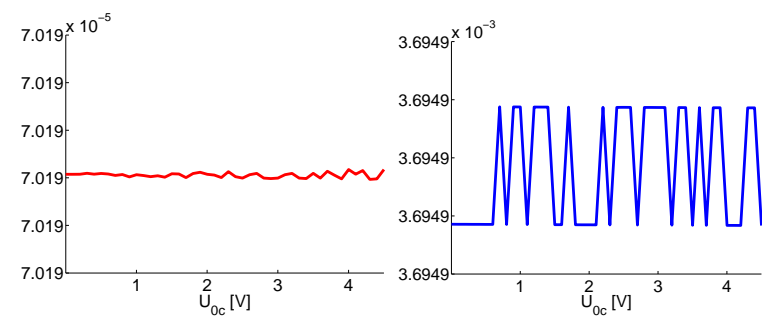

Fig. 4.4. Test 1: $\mathcal{L}^{2}$-error after the second Greedy step for the concentration (left) and the potential (right).
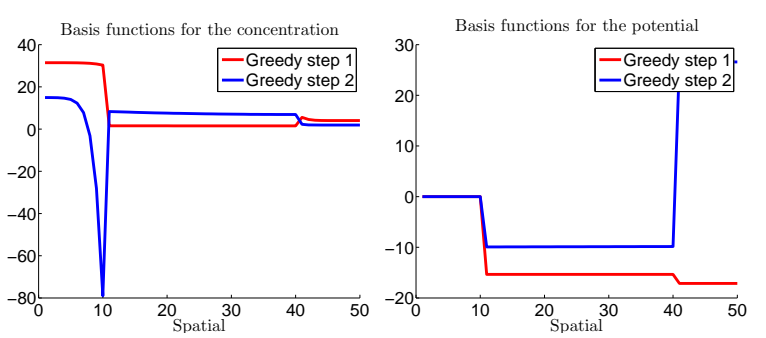

Fig. 4.5. Test 1: Basis functions for the first Greedy step for the concentration (left) and the potential (right).

negative electrode. We choose the following parameter set range: for the diffusion coefficients $D_{e c} \epsilon$ $\left[1.0 \cdot 10^{-9}, 1.1 \cdot 10^{-9}\right], \quad D_{e e} \in\left[7.5 \cdot 10^{-7}, 7.6 \cdot 10^{-7}\right]$,
$D_{e a} \in\left[3.9 \cdot 10^{-10}, 4.0 \cdot 10^{-10}\right]$, the transference number $t_{+} \in[0.2,0.3]$ and the reaction rates $k_{c}, k_{a} \in$ $[0.02,0.022]$. We discretize the parameter set in the following way: for the diffusion coefficients we choose the boundary values, for the other parameters we also take the boundary values and a value in between. With this discretization we get a 216-dimensional trainings set. All the other parameters are fixed like in table 4.1 noted, but in contrast to the previous subsection the POD tolerance is set $1-1 \cdot 10^{-8} \%$.

The graphical results are listed in the Figures 4.6 and 4.7: In Figure 4.6 the finite volume solutions for the first parameter set can be seen. The first parameter set is the one denoted in table 4.1. The $\mathcal{L}^{2}$-error of the reduced solutions in comparison to the finite volume solution is for the concentration smaller than $10^{-5}$ and for the electrical potential $10^{-4}$ to all 216 parameter sets. The $\mathcal{L}^{\infty}$-error is smaller than $10^{-3}$ for the concentration as well as for the potential after one Greedy step. The basis functions are plotted in Figure 4.7: there are four basis functions for the concentration and three for the potential.
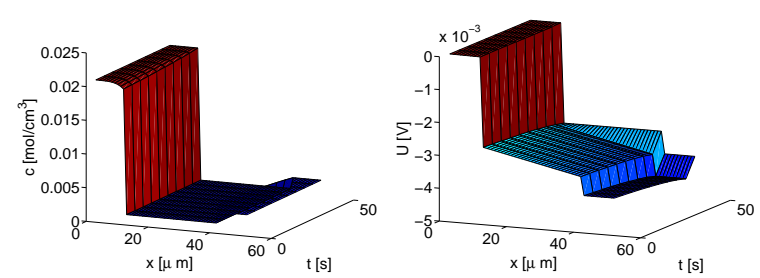

Fig. 4.6. Test 2: Finite volume solution fort the concentration (left) and the potential (right) for first parameter set
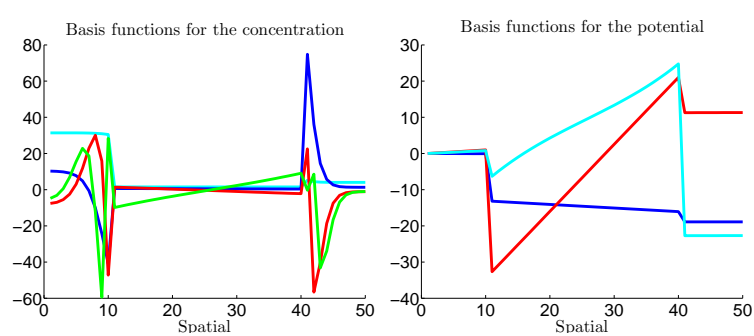

Fig. 4.7. Test 2: Basis functions after the first Greedy step for the concentration (left) and the potential (right).

The $\mathcal{L}^{2}$-error between the reduced solution and the true solution is after one Greedy step smaller than the $\mathcal{L}^{2}$-error tolerance $\epsilon_{\mathcal{L}^{2}}$ to all 216 parameter set. That means that just the information of one computational expensive true solution is needed to compute the reduced solutions to all 216 parameter sets with an acceptable error.

The speed up of the reduced solution in comparison to the true solution is 12.37 .

\section{Discussion}

In the present document we do the same approach like in [10].

The reduced basis approach works for the transport equation in a lithium-ion battery: in the above numerical 


\begin{tabular}{|c|c|}
\hline Notation & \\
\hline $\begin{array}{l}c,\left[\frac{m o l}{c m^{3}}\right] \\
\phi,[V]\end{array}$ & $\begin{array}{l}\text { concentration of the lithium/lithium-ions } \\
\text { electrical potential }\end{array}$ \\
\hline$\alpha,\left[\frac{\mathrm{cm}^{2}}{\mathrm{~s}}\right]$ & coefficient function \\
\hline$\beta,\left[\frac{\mathrm{mol}}{V \cdot \mathrm{cm} \cdot \mathrm{s}}\right]$ & coefficient function \\
\hline$\lambda,\left[\frac{A \cdot \mathrm{cm}^{2}}{\mathrm{~mol}}\right]$ & coefficient function \\
\hline$I,[A]$ & current \\
\hline$D_{e},\left[\frac{\mathrm{cm}^{2}}{\mathrm{~s}}\right]$ & diffusion coefficient \\
\hline$\kappa,\left[\frac{A^{S}}{V \cdot c m}\right]$ & electric/ionic conductivity \\
\hline$c^{0},\left[\frac{\mathrm{mol}}{\mathrm{cm}^{3}}\right]$ & $\begin{array}{l}\text { start concentration of lithium in the } \\
\text { electrodes/electrolyte }\end{array}$ \\
\hline$c_{\max },\left[\frac{\mathrm{mol}}{\mathrm{cm}^{3}}\right]$ & maximum of lithium the electrode can store \\
\hline$U_{0},[V]$ & open circuit potential \\
\hline$t_{+},[-]$ & transference number \\
\hline$k,\left[\frac{A}{c m^{2}}\right]$ & reaction rates \\
\hline$N .,[-]$ & number of control volumes \\
\hline$A .,\left[\mathrm{cm}^{2}\right]$ & cross section \\
\hline
\end{tabular}

TABLE 1.2

NOTATIONS OF THE BATTERY PARAMETERS

tests there are at most two Greedy steps needed to reach the $\mathcal{L}^{2}$-error tolerance $\epsilon_{\mathcal{L}^{2}}$. If the knowledge of all finite volume solutions to the training set is needed, the method would not be sufficient. In the above numerical tests we see that the limiting factor is the potential: for the concentration one Greedy step is sufficient but for the potential we need in some cases an additional Greedy step.

The application of the reduced basis method to this problem is not completed yet: We have to develop an a posteriori error estimator so that we do not have to compute all true solutions to the discretized parameter set. Further the computational time of the reduced solutions in comparison to the finite volume solutions for the presented numerical tests are fast but not so fast as it could be. In every Newton step we have to evaluate the nonlinearities completely. Also we have no affine parameter dependence. If you have a linear(ized) affine parameter dependent problem you can separate the parameter dependence from the bilinear form and from the linear form. To get an affine parameter dependent problem as well as a linearized problem we have to apply the (discrete) empirical interpolation method, cf. for instance [2], [3].

\section{ACKNOWLEDGMENTS}

The authors gratefully acknowledge support by the Adam Opel AG. Besides Competence Center The Virtual Vehicle (Graz) supported the lecture by Mr. Volkwein within the scope of the IGTE Symposium.

\section{APPENDIX}

\section{NOTATION}

In Table 1.2 one can find some notations for the battery parameters.

\section{REFERENCES}

[1] P.W. Atkins, "Physikalische Chemie", Wiley-VCH, 2., vollst. neubearb. A., 1996.

[2] M. Barrault and N.C. Nguyen and Y. Maday and A.T. Patera, "An "Empirical Interpolation" Method: Application to Efficient Reduced-Basis Discretization of Partial Differential Equations", C. R. Acad. Sci. Paris, Série I., pp. 667-672, 339, 2004.

[3] S. Chaturantabut and D.C. Sorensen, "Nonlinear Model Reduction via Discrete Empirical Interpolation”, SIAM J. Sci. Comput., 32(5), pp. 2737-2764, 2010.

[4] M. Doyle and T.F. Fuller and J. Newman, "Modeling of Galvanostatic Charge and Discharge of the Lithium/Polymer/Insertion Cell", Journal of The Electrochemical Society, 140(6), pp. 1526-1533, 1993.

[5] C. M Doyle, "Design and Simulation of Lithium Rechargeable Batteries", Ph.D. thesis, 1995.

[6] W. Dreyer and M. Gaberscek and C. Guhlke and R. Huth and J. Jamnik, "Phase Transition and Hysteresis in a Recharchable Lithium Battery Revisited", European J. Appl. Math., 22, pp. 267 290, 2011.

[7] A.-L. Gerner and K. Veroy, "Certified reduced basis method for parameterized saddle point problems", SIAM J. Sci. Comput., (accepted Jul 2012).

[8] M.A. Grepl and A.T. Patera, "A Posteriori Error Bounds for Reduced-Basis Approximations of Parametrized Parabolic Partial Differential Equations", Mathematical Modelling and Numerical Analysis, 2005, 39(1), pp. 157-181.

[9] M.A. Grepl, Y. Maday, N.C. Nguyen, and A.T. Patera, "Efficient Reduced-Basis Treatment of Nonaffine and Nonlinear Partial Differential Equations", Mathematical Modelling and Numerical Analysis, 2007, 41(3), pp. 575-605.

[10] B. Haasdonk and M. Ohlberger, "Reduced Basis Method for Finite Volume Approximations of Parametrized Linear Evolution Equations", Math. Model. Numer. Anal., 42(2), 2008, pp. 277-302.

[11] P. Holmes and J.L. Lumley and G. Berkooz and C. Rowley, "Turbulence, Coherent Structures, Dynamical Systems and Symmetry", Cambridge Monographs on Mechanics, 2012, Cambridge University Press.

[12] O. Lass and S. Volkwein, "POD Galerkin schemes for nonlinear elliptic-parabolic systems", submitted, 2011.

[13] A. Latz and J. Zausch and O. Iliev, "Modeling of Species and Charge Transport in Li-Ion Batteries Based on Non-Equilibrium Thermodynamics", Lecture Notes in Computer Science 6046, 329337, 2011.

[14] A. Latz and J. Zausch, "Thermodynamic Consistent Transport Theory of Li-Ion Batteries", Journal of Power Sources 196, 32963302, 2011.

[15] J. S. Newman and K. E. Thomas-Alyea, "Electrochemical Systems", Wiley John + Sons, 3rd ed., 2004.

[16] A. T Patera and G. Rozza, Reduced Basis approximation and A Posteriori Error Estimation for Parametrized Partial Differential Equations, MIT, 2007.

[17] P. Popov, Y. Vutov, S. Margenov and O. Iliev, "Finite Volume Discretization of Equations Describing Nonlinear Diffusion in LiIon Batteries," Fraunhofer ITWM report 191, 2010.

[18] S. Volkwein, "Model Reduction Using Proper Orthogonal Decomposition", lecture notes, Konstanz, 2011.

[19] J. Wu and J. Xu and H. Zou, "On the Well-posedness of a Mathematical Model for Lithium-Ion Battery Systems, Methods and Applications of Analysis, 13(3), pp. 275-298, 2006. 